\title{
Tela de Amsler e campo visual no rastreamento da retinopatia por cloroquina
}

\author{
Amsler grid and visual field on screening for chloroquine retinopathy
}

Bruno Vargas Madalena ${ }^{1}$, Akiyoshi Oshima², Pedro Durães Serracarbassa ${ }^{2}$

\section{RESUMO}

Objetivos: Comparar a tela de Amsler modificada com o campo visual Humphrey ${ }^{\circledR}$ 10-2 vermelho nos usuários de cloroquina, na detecção da maculopatia precoce, e correlacionar com as variáveis de risco.

Métodos: Foram analisados 116 olhos de 58 pacientes, acompanhados no Serviço de Oftalmologia do Hospital do Servidor Público Estadual de São Paulo, entre abril de 2006 e abril de 2008. Todos os usuários tinham fundo de olho normal e mais de dois anos de terapia com cloroquina. Os participantes tiveram seus dados clínicos avaliados e foram submetidos ao exame da acuidade visual corrigida, biomicroscopia de fundo, campimetria macular automatizada e tela de Amsler.

Resultados: A incidência da maculopatia precoce foi de 7 a 10\%, dependendo do exame considerado. A concordância entre a tela de Amsler e o campo visual foi baixa. Para o grupo de olhos que apresentaram ambos os exames alterados, houve significância estatística com a alta dose diária, dose cumulativa elevada e baixa acuidade visual; a idade do paciente e a duração do tratamento não mostraram boa correlação nestes casos, mas suas médias (67,4 anos e 8,4 anos, respectivamente) situaram-se dentro da faixa dos fatores de alto risco.

Conclusões: $O$ estudo sugere que a tela de Amsler pode ser útil na complementação das informações do campo visual no rastreamento periódico da retinopatia por cloroquina, sobretudo naqueles com fatores de alto risco bem estabelecidos, selecionando melhor os candidatos à realização de testes objetivos, como o OCT de alta resolução e o ERG multifocal.

Descritores: Cloroquina/efeitos adversos; Retina/efeito de drogas; Rastreamento; Campos visuais; Acuidade visual; Fatores de risco

\begin{abstract}
Purpose: To compare the modified Amsler grid to the Humphrey ${ }^{\circledR} 10-2$ red visual field in chloroquine users for the detection of early maculopathy, and to correlate with the risk variables.

Methods: The study included 116 eyes of 58 patients followed at the Department of Ophthalmology of Hospital do Servidor Público Estadual de São Paulo, from April, 2006 to April, 2008. All users had normal fundus and more than 2 years of chloroquine therapy. Their clinical data were evaluated and they underwent visual acuity examination, fundus biomicroscopy, visual field and Amsler grid.

Results: The incidence of early maculopathy was 7 to 10\%, depending on the examination considered. The agreement between the Amsler grid and visual field was low. There was statistical significance with the use of high daily dose, elevated cumulative dose and low visual acuity in patients whose eyes had both abnormal tests; patient age and duration of treatment did not show good correlation in these cases, but their averages (67.4 years and 8.4 years, respectively) were within the range of high risk factors.

Conclusions: The study suggests that Amsler can be useful in complementing the information on the visual field for chloroquine retinopathy periodic screening, especially for those patients who present high risk factors well established, selecting better candidates for objective tests, such as HDOCT and MfERG.
\end{abstract}

Keywords: Chloroquine/adverse effects; Retina/drug effects; Screening; Visual fields; Visual acuity; Risk factors

\section{INTRODUÇÃO}

O difosfato de cloroquina (DFC) e o sulfato de hidroxicloroquina (SHC) são fármacos eficientes e utilizados há várias décadas no tratamento de inúmeras doenças do tecido conectivo, sendo amplamente utilizados até a atualidade ${ }^{(1)}$. Ambos podem apresentar, porém, toxicidade retiniana ${ }^{(2)}$. O DFC é duas a três vezes mais tóxico para a retina do que o $\mathrm{SHC}^{(3)}$. Podem causar degeneração do epitélio pigmentado retiniano e destruição dos fotorreceptores e células ganglionares ${ }^{(4)}$, resultante da inibição de fosfolipases lisossomais e síntese proteica(5). Há perda da visão central como queixa principal.

A "maculopatia precoce" por cloroquina é definida pela presença de escotomas relativos, bilaterais e reprodutíveis, detectados por um dos métodos perimétricos teste com tela de Amsler (Amsler) ou teste de campo visual automatizado 10 graus com estímulo vermeIho (CV); a "maculopatia estabelecida" é representada por escotomas absolutos bilaterais, detectados por ambos os exames, que já indicam alterações fundoscópicas ${ }^{(2)}$.
Os fatores de risco para toxicidade retiniana por DFC incluem dose diária maior que $250 \mathrm{mg} / \mathrm{dia}^{(4)}$ ou maior que $3 \mathrm{mg} / \mathrm{kg} /$ dia por mais de 5 anos $^{(6)}$ ou dose cumulativa maior que $300 \mathrm{~g}^{(4)}$, insuficiência hepática e/ou renal, obesidade (devido hiperdosagem), doença retiniana prévia e idade maior que $60 \operatorname{anos}^{(6)}$. A dose diária é considerada o fator mais relevante ${ }^{(7,8)}$.

A cloroquina deverá ser suspensa ou diminuída após o diagnóstico de maculopatia(9,10). É fundamental atentar-se aos defeitos precoces do campo visual central, pois as alterações ainda podem ser reversíveis ${ }^{(11)}$

Um exame oftalmológico de base deve ser realizado no início da terapia ${ }^{(6)}$. Posteriormente, exames anuais de rotina devem incluir acuidade visual, fundoscopia e teste com tela de Amsler e/ou CV(12,13). O CV vermelho é muito mais sensível (91\%) do que o branco, mas com especificidade menor $(58 \%)^{(2)}$. A tela de Amsler com linhas brancas em fundo preto (convencional) é mais específica que a com linhas vermelhas, pois esta apresenta muitos falsos positivos ${ }^{(14)}$. 
A angiofluoresceinografia ${ }^{(9,15)}, \mathrm{o}$ teste de Ishihara $(11,15,16)$, a eletrooculografia, o eletrorretinograma (ERG) difuso $(2,9,15,17)$ e a tomografia de coerência óptica (OCT) por domínio de tempo não detectam a doença na fase pré-clínica. O teste de sensibilidade ao contraste ${ }^{(6,18)}$ pode ser útil na detecção precoce. Recente revisão das recomendações da Academia Americana de Oftalmologia aponta para o rastreamento com novas tecnologias, quando acessíveis ao médico, tais como OCT de alta resolução, autofluorescência retiniana e ERG multifocal, pois parecem ser mais precoces que os exames perimétricos ${ }^{(5,6,15,19)}$. O mesmo artigo condena o uso do Amsler, uma vez que despende muita atenção para uma leitura correta, mas já há controvérsias(20).

Há autores que defendem a falta de necessidade de acompanhamento(18,21,22), mas novos casos não publicados em todo o mundo apontam para a importância do diagnóstico precoce ${ }^{(23)}$.

Este estudo teve por objetivos comparar as alterações da tela de Amsler modificada às alterações do CV nos usuários de DFC, para avaliar o melhor método de detecção da maculopatia precoce (retinopatia inicial medicamentosa em pacientes que ainda não apresentam alterações fundoscópicas) e correlacionar as variáveis idade, tempo de uso do fármaco, dose diária, dose cumulativa e acuidade visual com os exames normais e alterados.

\section{MÉTODOS}

Neste estudo seccional, foram atendidos 58 pacientes (116 olhos), no Serviço de Oftalmologia do Hospital do Servidor Público Estadual SP, entre abril de 2006 e abril de 2008. O projeto teve aprovação do Comitê de Ética em Pesquisa do HSPE-SP (Projeto número 0032.0.338.000-07) e todos os pacientes assinaram termo de consentimento informado individual.

Para o cálculo do tamanho amostral, foi considerada a população finita dos usuários de DFC do Serviço de Reumatologia do referido hospital, com erro amostral de 9\%, considerando a incidência da retinopatia igual a 15\%.

Os critérios de inclusão englobaram usuários de DFC há pelo menos dois anos, que estavam sendo acompanhados no ambulatório de doenças do segmento posterior. Os pacientes foram submetidos à avaliação de acuidade visual corrigida (com a tabela de Snellen, a 6 metros de distância e transformada em logMAR), biomicroscopia anterior e de fundo, medida de pressão intraocular, CV Humphrey ${ }^{\circledR}$ (Carl Zeiss Meditec, Inc) 10-2 (com mira vermelha e estímulo III, estratégia limiar total) e de teste com tela de Amsler modificada (linhas pretas em fundo branco(24), a $35 \mathrm{~cm}$ de distância, sob boa luminosidade). Estes pacientes foram submetidos a nova consulta, com intervalo médio de 18 semanas, na qual todo o exame clínico e os exames perimétricos foram repetidos, para confirmação dos resultados obtidos na primeira avaliação. Todos deveriam apresentar fundo de olho normal durante o seguimento. Os testes de CV e com a tela de Amsler foram aplicados em sequência aleatória.

Foram excluídos aqueles que apresentaram ametropias significativas (maiores que 5 dioptrias), alterações corneanas importantes, opacidade moderada de meios e qualquer maculopatia prévia. Exames de CV cujos índices revelaram baixa confiança ou "efeito aprendizagem"também foram desprezados.

Os exames foram considerados anormais quando: 1) CV mostrou presença de dois ou mais pontos paracentrais adjacentes com queda de $5 \mathrm{~dB}$ em relação à referência central (fóvea); 2) Amsler apresentou manchas ou linhas paracentrais apagadas ou tortuosas.

Foi analisada a frequência de exames alterados (com os respectivos intervalos de confiança de 95\%) quando se considerou alteração: 1) somente no $\mathrm{CV}$; 2) somente no Amsler; 3 ) em qualquer exame (Amsler e/ou CV); 4) nos dois exames simultaneamente (Amsler e CV).

A existência de associação entre os dois testes foi verificada com uso do teste exato de Fisher. A concordância entre eles foi estudada com o coeficiente kappa.
As variáveis de risco (idade, acuidade visual, tempo total de uso da cloroquina, dose diária e cumulativa da droga) foram descritas com a utilização de medidas-resumo (média, mediana, mínimo, máximo e desvio padrão), e foram correlacionadas com os grupos de pacientes com exames alterados. Para a análise utilizou-se o teste não paramétrico de Mann-Whitney.

Para os testes foi considerado nível de significância de 5\%.

\section{RESULTADOS}

A média da idade dos participantes foi de 60,34 $\pm 12,22$ anos (variando entre 24 e 85 ). O tempo total médio de terapia foi de $5,39 \pm 3,54$ anos (variando de 2 a 18). A média da dose diária foi de $218,89 \pm 65,12$ mg (variando de 125 a 400), enquanto a média da dose cumulativa, de 477,42 $\pm 395,40 \mathrm{~g}$ (variando de 91 a 1642). A média da acuidade visual transformada em logMAR foi de 0,13 \pm 0,24 (variando de 1,16 a -0,04, ou de 20/300 a 20/18).

A tabela 1 evidenciou que a maior frequência de alteração entre os dois exames ocorreu no CV (15,5\%); para olhos anormais em qualquer exame, a frequência foi consequentemente maior $(25,0 \%)$; e apenas 5,2\% dos olhos apresentaram alteração simultânea em ambos os exames.

$\mathrm{Na}$ tabela 2, houve associação entre ambos os exames pelo teste exato de Fisher ( $p=0,025)$, porém a concordância entre eles foi baixa, uma vez que o valor do kappa ficou próximo de zero $(0,226)$.

Quando se considerou a presença de alterações em ambos os testes e se correlacionou com as diferentes variáveis de risco, encontrou-se médias de: 59,7 e 67,4 anos para idade, 5,2 e 8,4 anos para tempo total de uso do fármaco, 211 e 300 mg para dose diária, 432 e 949 g para dose cumulativa e de 0,12 e 0,25 para acuidade visual logMAR, respectivamente nos grupos de pacientes com exames normais e alterados. A diferença das médias foi estatisticamente significativa para dose diária $(p=0,021)$, dose cumulativa $(p=0,012)$ e acuidade visual $(p=0,034)$.

Tabela 1. Frequência dos olhos alterados segundo avaliação do campo visual, da tela de Amsler, de qualquer exame e de ambos conjuntamente, com os respectivos intervalos de confiança (95\%)

\begin{tabular}{lcccc}
\hline & & & \multicolumn{2}{c}{ IC (95\%) } \\
\cline { 4 - 5 } Variável & Frequência & \% & Inferior & Superior \\
\hline CV & 18 & 15,5 & 8,9 & 22,1 \\
Amsler & 17 & 14,7 & 8,2 & 21,1 \\
CV e/ou Amsler & 29 & 25,0 & 17,1 & 32,9 \\
CVe Amsler & 6 & 5,2 & 1,1 & 9,2 \\
\hline Total & 116 & 100 & &
\end{tabular}

$\mathrm{CV}=$ campo visual; $\mathrm{IC}=$ intervalo de confiança

Tabela 2. Existência de associação e medida de concordância entre os resultados do campo visual e da tela de Amsler, por olho

\begin{tabular}{|c|c|c|c|c|c|c|c|c|}
\hline \multirow[b]{3}{*}{ CV } & \multicolumn{4}{|c|}{ Amsler } & & & \multirow[b]{3}{*}{ kappa } & \multirow[b]{3}{*}{ pFishe } \\
\hline & \multicolumn{2}{|c|}{ Normal } & \multicolumn{2}{|c|}{ Alterado } & \multicolumn{2}{|c|}{ Total } & & \\
\hline & $n$ & $\%$ & $\mathbf{n}$ & $\%$ & $n$ & $\%$ & & \\
\hline Normal & 87 & 75,0 & 11 & 9,5 & 98 & 84,5 & 0,226 & 0,025 \\
\hline Alterado & 12 & 10,3 & 6 & 5,2 & 18 & 15,5 & & \\
\hline Total & 99 & 85,3 & 17 & 14,7 & 116 & 100 & & \\
\hline
\end{tabular}

$\mathrm{CV}=$ campo visual; $\mathrm{n}=$ número de olhos; $\mathrm{kappa}=$ coeficiente $k a p p a$;

pFisher= nível de significância para teste exato de Fisher

* = teste exato de Fisher e de concordância kappa 
Quando se considerou a presença de alterações em qualquer exame, os grupos de pacientes com exames normais e alterados apresentaram médias de idade e de acuidade visual de 56,97 e 66,75 anos, e de 0,09 e 0,23, respectivamente, com diferenças estatisticamente significativas entre os grupos. Quando se considerou alterações somente no CV, a diferença entre as médias da idade, dose diária e acuidade visual nos grupos normal e alterado foi estatisticamente significativa. Para o grupo com alterações somente no Amsler, não houve boa correlação entre exames normais e alterados.

\section{DISCUSSÃO}

Uma vez que não há consenso sobre um teste padrão para rastreamento da retinopatia por DFC em fase pré-clínica, o presente estudo avaliou somente a porcentagem de exames alterados em pacientes usuários da droga, impossibilitando o cálculo de sensibilidade e especificidade dos testes de CV e com tela de Amsler.

O tamanho amostral calculado para o estudo foi de 56 participantes, dentre cerca de 1000 pacientes usuários ou ex-usuários de DFC acompanhados no Serviço de Reumatologia do HSPE-SP. Este cálculo levou em consideração apenas 660 integrantes desta população, já que na prática clínica considera-se que aproximadamente 1/3 deles abandonam o tratamento.

O CV utilizado foi aquele com mira vermelha, em contraposição às novas recomendações americanas ${ }^{(19)}$, já que é mais sensível do que específico ${ }^{(11)}$, mais adequado ao rastreamento de uma doença rara e ainda em fase pré-clínica, dando-se maior ênfase à capacidade de detecção do exame. A tela utilizada foi a de fundo branco "Amsler modificada", uma vez que é predominante nos materiais oftalmológicos (pela facilidade de impressão) e não apresenta diferenças significativas em relação à original quando testada em pacientes com acuidade visual maior do que 20/40 (0,3 logMAR) $)^{(24)}$, coincidindo com as médias dos grupos estudados.

Não houve pacientes hepatopatas e/ou nefropatas neste estudo.

Observou-se que a média de idade dos pacientes com exames alterados foi maior que 60 anos, coincidindo com o grupo de risco apontado na literatura ${ }^{(6,25)}$, independentemente se houve significância estatística da diferença de idade entre aqueles com testes alterados ou normais; a média de idade dos pacientes com exames normais foi abaixo de 60 anos.

O tempo total de uso da droga, apesar de não ter sido uma variável com diferença estatisticamente significante, fato igualmente encontrado em outros trabalhos ${ }^{(21,26)}$, teve sua média notavelmente mais alta no grupo de pacientes com ambos exames alterados, sendo maior que 5 anos em todos os grupos alterados, concordando com a definição dos fatores de alto risco ${ }^{(6,25)}$.

A dose diária total foi significativamente maior no grupo com alterações no CV e sobretudo naquele com ambos exames anormais, com médias superiores a $250 \mathrm{mg} / \mathrm{dia}$. No grupo com Amsler ou qualquer dos dois exames alterados, não houve boa relação entre os pacientes normais e os alterados, e os níveis foram inferiores a 250 $\mathrm{mg} / \mathrm{dia}$. Este achado coincide com os dados encontrados na literatura, que relata que pacientes com doses diárias elevadas têm maior risco de desenvolverem a maculopatia precoce comprovada pelos exames complementares ${ }^{(2,6,8,23)}$, caso daqueles que administram mais que $250 \mathrm{mg} / \mathrm{dia}^{(4)}$ (concentração considerada segura na prática clínica, correspondente a 1 comprimido comercial(6)). Entretanto, muitos autores não consideram a dose diária total, mas calculam a dose por "peso magro"(25), já que um comprimido de 250 mg por dia pode ser uma superdosagem aos pacientes com menos de $83 \mathrm{~kg}$ de peso(6) (sobretudo nas mulheres, representantes da grande maioria deste estudo) e $3 \mathrm{mg} / \mathrm{kg} /$ dia também pode ser uma dose excessiva para uma pessoa obesa (uma vez que a cloroquina não se acumula no tecido adiposo $\left.{ }^{(6)}\right)$. Vale lembrar, contudo, que novo consenso na literatura estrangeira reconsidera a dose diária total como a mais adequada para fator de risco, exceto em indivíduos brevilíneos ${ }^{(19)}$.
A dose cumulativa não guardou relação com os exames alterados isoladamente, mesmo achado encontrado por outros autores ${ }^{(21,26)}$, mas foi significativamente maior nos pacientes com ambos exames alterados. A dose cumulativa média de todos os grupos, com alterações ou não, foi superior a 430 g, sugerindo que o nível de 300 g, antes considerado seguro para a droga ${ }^{(4)}$, pode estar abaixo do limiar de risco observado na prática clínica, como já estabelecido em novas diretrizes internacionais ${ }^{(19)}$, que refere dose cumulativa maior que 460 gramas como um fator de risco a ser considerado.

Neste estudo, a média da acuidade visual não apresentou diferença significativa entre olhos normais e alterados para Amsler isoladamente, mas foi estatisticamente menor nos demais grupos alterados, sobretudo naquele com alterações para CV isoladamente $(p=0,001)$, permitindo inferir que o CV é mais influenciado pela baixa visão, discordando do achado de outros trabalhos ${ }^{(15,16)}$. Ressalva-se, no presente estudo, 1 caso de catarata inicial unilateral que pode ter causado baixa acuidade visual no olho correspondente, induzindo viés na análise.

Na revisão da literatura mundial, há relatos de incidência da retinopatia por SHC entre 0 e 4\%(1); para o DFC, porém, a incidência é muito inconstante entre os autores, variando de quase nula a mais de 40\%(11) (média de 15\%), a depender da definição utilizada para enquadramento do estágio da doença. Alguns estudos revelaram incidência da maculopatia por DFC acima de 20 vezes à da SHC(2).

Recente trabalho brasileiro registrou prevalência da maculopatia por DFC de $38,9 \%$ se consideradas alterações no CV, e de $5,55 \%$ se consideradas alterações na tela de Amsler ${ }^{(26)}$. No presente estudo, segundo a definição de Easterbrook, a maculopatia precoce (alterações bilaterais discriminadas por 1 exame perimétrico) foi apontada por $6,9 \%$ dos pacientes, de acordo com análise do CV, e por 10,3\% deles, se considerado o exame de Amsler; mas apenas 1 paciente $(1,72 \%)$ teve a maculopatia estabelecida (alterações bilaterais em ambos exames), representada por usuário de longa data com dose diária elevada (375 mg/dia por 10 anos). Esta comparação mostrou importante divergência na frequência dos exames anormais entre os estudos, embora naquele o tempo total de terapia e a dose cumulativa foram maiores, tendo sido incluídos também os pacientes com fundoscopia alterada.

Quando se considerou cada olho isoladamente, houve 15,5\% de exames alterados no CV e 14,7\% de exames alterados na tela de Amsler. Um quarto $(25,0 \%)$ dos olhos analisados tiveram alterações se considerado qualquer exame, mas apenas $5,2 \%$ deles foram anormais para ambos os testes ou seja, somente $1 / 5$ dos olhos alterados para qualquer exame foram também para ambos.

Em contraposição à definição de maculopatia precoce e reversível, que cita alterações bilaterais ${ }^{(11)}$, foram também considerados exames alterados aqueles unilaterais, para efeito de rastreamento. Isto se justifica pelo fato de que, no Brasil, diferentemente dos países mais desenvolvidos, a grande maioria dos usuários de cloroquina a utilizam sob a forma de difosfato, mais barata e também mais tóxica, cujo potencial de dano ocular é maior se o acompanhamento não for mais rigoroso. Este trabalho, portanto, não considerou relevante apenas as alterações bilaterais, mas sim aquelas presentes unilateralmente para ambos exames enquadrando-se em uma "possível" retinopatia precoce assimétrica, para a qual já é aconselhável a diminuição ou interrupção da terapia, desde que esta não seja essencial à afecção de base ${ }^{(6)}$.

Neste ponto de vista, a frequência dos pacientes que tiveram ao menos 1 olho alterado para ambos exames foi de 5 (8,62\%); destes, apenas $1(1,72 \%)$ teve os dois olhos anormais aos dois testes. Este grupo apresentou tanto idade, como tempo total de uso, dose diária e cumulativa notavelmente maiores que das demais pessoas; analogamente, a acuidade visual deste grupo foi menor que a dos outros (as três últimas variáveis tiveram significância estatística).

A concordância entre CV e Amsler é um achado muito inconstante entre os autores, variando de baixa ${ }^{(26)}$ a alta $^{(16)}$. Como neste trabalho 
houve baixa relação entre os exames, sendo observado que apenas um terço dos olhos anormais para um teste também foi alterado para o outro, e ainda não foi estabelecido um teste padrão para o rastreamento da maculopatia precoce(26), este estudo permitiu a conclusão de que Amsler e CV foram exames não substitutivos, mostrando que Amsler, um teste simples, barato e autoaplicável pelo paciente, pode ser útil na complementação das informações do CV. Ambos podem ser realizados tanto na admissão quanto no acompanhamento semestral dos usuários ou ex-usuários do DFC, mesmo naqueles sob doses diária e cumulativa seguras (grupos de baixo risco).

Vale lembrar que atual consenso ${ }^{(19)}$ adota para o rastreamento da maculopatia precoce somente o CV, como exame subjetivo, e ao menos um dos exames objetivos modernos, seja funcional - eletrorretinograma multifocal|(5,19,25), quanto estrutural OCT de alta resolução e autofluorescência ${ }^{(19)}$, por serem mais precoces que o CV e Amsler. Entretanto, por serem de alto custo e com acesso restrito a grandes centros, teriam uma melhor indicação quando o CV se tornasse alterado, e os achados adicionais da tela de Amsler poderiam auxiliar na escolha destes candidatos.

\section{CONCLUSÕES}

Houve baixa concordância entre CV e Amsler, sugerindo que os mesmos não são substitutivos, sendo Amsler mais apropriado na análise conjunta com CV.

Observou-se que as alterações presentes em qualquer exame foram excessivamente frequentes, e portanto não foram valorizadas; quando apontadas por ambos os testes, porém, foram consideradas como significativas e tiveram boa correlação com o aumento de outras variáveis, tais como dose diária e cumulativa, e com a diminuição da acuidade visual (fatores de alto risco para o desenvolvimento da doença). Foi verificado que o grupo com ambos exames alterados teve a dose diária média maior que 250 mg, e cumulativa maior que $460 \mathrm{~g}$, consideradas como principais fatores de risco para o desenvolvimento da afecção. Estes usuários tiveram a administração da droga interrompida ou diminuída, mesmo nos exames alterados unilateralmente, já que o DFC tem maior toxicidade ocular do que a hidroxicloroquina.

\section{REFERÊNCIAS}

1. Yam JC, Kwok AK. Ocular toxicity of hydroxychloroquine. Hong Kong Med J. 2006 12(4):294-304.

2. Easterbrook M. Detection and prevention of maculopathy associated with antimalarial agents. Int Ophthalmol Clin. 1999;39(2):49-57.

3. Rynes RI. Ophthalmologic safety of long-term hydroxychloroquine sulfate treatment Am J Med. 1983;75(1A):35-9.
4. Gouveia EB, Morales MSA, Gouveia GB, Lourenzi VPM. Toxicidade ocular por derivados da 4-aminoquinolona. Arq Bras Oftalmol. 2007;70(6):1046-51.

5. Ma X, Yan L, He L, He D, Lu H. Ocular fundus manifestation of two patients following long-term chloroquine therapy: a case report. Diagn Pathol. 2010;5:20.

6. Marmor MF, Carr RE, Easterbrook M, Farjo AA, Mieler WF; American Academy of Ophthalmology. Recommendations on screening for chloroquine and hydroxychloroquine retinopathy: a report by the American Academy of Ophthalmology. Ophthalmology. 2002;109(7):1377-82.

7. Levy GD, Munz SJ, Paschal J, Cohen HB, Pince KJ, Peterson T. Incidence of hydroxychloroquine retinopathy in 1,207 patients in a large multicenter outpatient practice. Arthritis Rheum. 1997;40(8):1482-6.

8. Bernstein HN. Ocular safety of hydroxychloroquine. Ann Ophthalmol. 1991;23(8):292-6.

9. Easterbrook M. The ocular safety of hydroxychloroquine. Semin Arthritis Rheum. 1993; 23(2 Suppl 1):62-7.

10. Weiner A, Sandberg MA, Gaudio AR, Kini MM, Berson EL. Hydroxychloroquine retinopathy. Am J Ophthalmol. 1991;112(5):528-34.

11. Easterbrook M. Ocular effects and safety of antimalarial agents. Am J Med. 1988; 85(4A):23-9.

12. Hart WM Jr, Burde RM, Johnston GP, Drews RC. Static perimetry in chloroquine retinopathy. Perifoveal patterns of visual field depression. Arch Ophthalmol. 1984; 102(3):377-80.

13. Easterbrook M, Trope G. Value of Humphrey perimetry in the detection of early chloroquine retinopathy. Lens Eye Toxic Res. 1989;6(1-2):255-68.

14. Lee AG. Hydroxychloroquine screening. Br J Ophthalmol. 2005;89(5):521-2.

15. Easterbrook M. The sensitivity of Amsler grid testing in early chloroquine retinopathy. Trans Ophthalmol Soc U K. 1985;104(Pt 2):204-7.

16. Easterbrook M. The use of Amsler grids in early chloroquine retinopathy. Ophthalmology. 1984;91(11):1368-72.

17. Bishara SA, Matamoros N. Evaluation of several tests in screening for chloroquine maculopathy. Eye (Lond). 1989;3(Pt 6):777-82.

18. Grierson DJ. Hydroxychloroquine and visual screening in a rheumatology outpatient clinic. Ann Rheum Dis. 1997:56(3):188-90.

19. Marmor MF, Kellner U, LaiTY, Lyons JS, MielerWF; American Academy of Ophthalmology. Revised recommendations on screening for chloroquine and hydroxychloroquine retinopathy. Ophthalmology. 2011;118(2):415-22

20. Flach AJ. Amsler grids for chloroquine toxicity. Ophthalmology. 2011;118(10):2099; author reply 2099-100. Comment on Ophthalmology. 2011;118(2):415-22.

21. Blyth C, Lane C. Hydroxychloroquine retinopathy: is screening necessary? BMJ. 1998; 316(7133):716-7. Comment in BMJ. 1998;317(7169):1388-9.

22. Morsman CD, Livesey SJ, Richards IM, Jessop JD, Mills PV. Screening for hydroxychloroquine retinal toxicity: is it necessary? Eye (Lond). 1990;4(Pt 4):572-6.

23. Browning DJ. Hydroxychloroquine and chloroquine retinopathy: screening for drug toxicity. Am J Ophthalmol. 2002;133(5):649-56. Comment in Am J Ophthalmol. 2002; 134(6):935; author reply 935-6.

24. Augustin AJ, Offermann I, Lutz J, Schmidt-Erfurth U, Tornambe P. Comparison of the original Amsler grid with the modified Amsler grid: result for patients with age-related macular degeneration. Retina. 2005;25(4):443-5.

25. Easterbrook M. Screening for antimalarial toxicity: current concepts. Can J Ophthalmol. 2002;37(6):325-8, 331-4.

26. Rodrigues LD, Shinjo SK, Oyamada MK, Serracarbassa PD, Takahashi WY, Borba EF, et al. Métodos diagnósticos para retinopatia induzida pelo difosfato de cloroquina nos portadores de lúpus eritematoso sistêmico. Arq Bras Oftalmol. 2009;72(3):313-20.
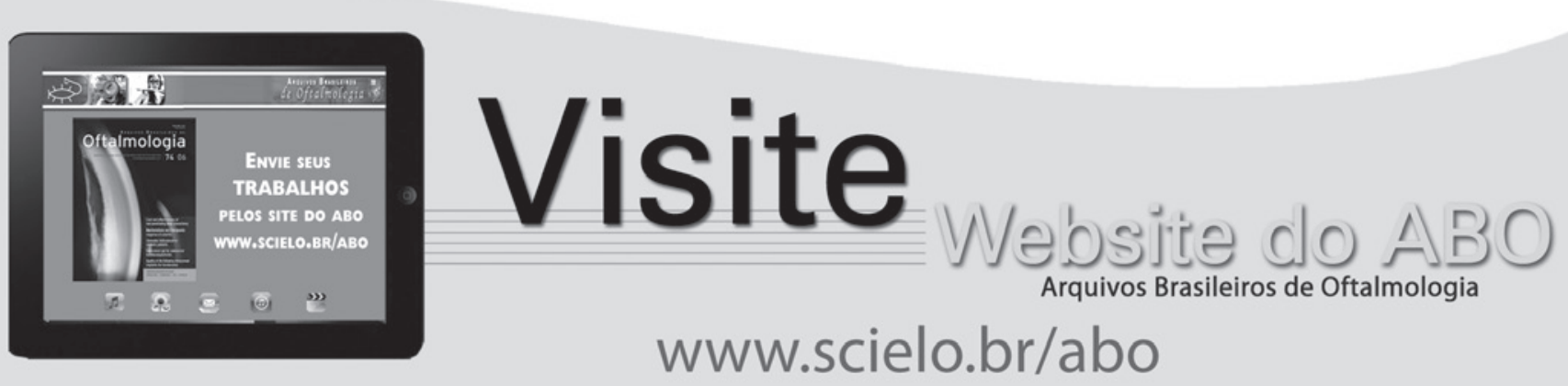